\title{
THEORY AND REALITY OF BEAM-BEAM EFFECTS AT HADRON COLLIDERS*
}

\author{
Y. Alexahin, FNAL, Batavia, IL 60510
}

\section{Abstract}

The beam-beam phenomena in hadron colliders are just as rich as in e+e- machines: orbit and focusing perturbations, excitation of nonlinear resonances, coherent tuneshifts. Moreover, the absence of radiation damping and long duration of a store permit even high-order (and correspondingly weak) resonances to manifest themselves presenting a major challenge for both theoretical analysis and machine operation. The recent progress in understanding of and coping with the beam-beam effects at hadron colliders, primarily at the Tevatron, is discussed.

\section{INTRODUCTION}

A good progress has been made in both theoretical and experimental study of beam-beam effects in hadron colliders since the advent of the first one, ISR. Major manifestations of the beam-beam effect - such as orbit offset, tunespread, resonance excitation - are well described analytically, reproduced in tracking simulations.

However, the overall effect of these factors on the time scale of many hours is more difficult to predict.

Somewhat surprising are relatively high losses (especially from the strong beam in the weak-strong case $[1,2])$ which are apparently produced by high-order beam-beam resonances: the width of these resonances and their synchrotron satellites is insufficient to overlap and cause dynamical stochasticity. It is important to understand the detail mechanism of these losses to make projections for new machines and upgrade of the existing ones.

Another important and not completely resolved issue is stability of coherent beam-beam modes. Existence of such

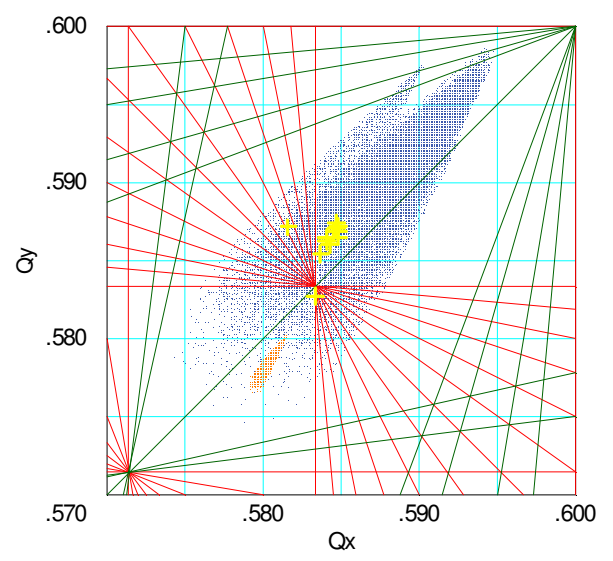

Figure 1. Tune distribution of protons (orange) and antiprotons (blue) in Tevatron.

* Work supported by the U.S. Department of Energy under contract No.DE-AC02-76CH03000 modes in colliding proton beams was experimentally demonstrated at RHIC [3]; recently there were observations of instability of coupled motion of $\mathrm{Cu}$ beams at injection energy [4]. At Tevatron, which is still running in the weak-strong regime (intensity ratio $0.1 \div 0.2$ ), attempts to significantly reduce chromaticity during collisions result in coherent beam-beam instability.

Observations at RHIC and Tevatron indicate that there is an intricate interplay between beam-beam and beamwall interactions which requires further investigation.

In the present report we discuss the current understanding of these problems and the efforts to overcome them.

\section{INCOHERENT BEAM-BEAM EFFECTS}

Absence of radiation damping in hadron colliders makes even high-order resonances dangerous, limiting available tune space. So far the largest beam-beam tuneshift, $\xi=0.025$, has been achieved at Tevatron.

Fig.1 shows typical Tevatron beam-beam footprint (store 3678, 07/28/04). Yellow crosses mark antiproton tunes (average for three bunches occupying the same positions in the trains) measured with $1.7 \mathrm{GHz}$ Schottky monitor [5]. Proximity to the main diagonal does not imply absence of coupling, it is just a result of pickups seeing both modes of transverse oscillations. Orange and blue dots present theoretically reconstructed tune distribution of on-momentum protons and antiprotons (coupling ignored, only one p-bunch shown).

In this particular store the intensities were not high $\left(N_{p}=2.5 \cdot 10^{11} / \mathrm{bunch}, N_{a}=3.5 \cdot 10^{10} / \mathrm{bunch}\right)$, in the result the on-momentum protons did not reach the $12^{\text {th }}$ order resonances while the antiprotons were below the $5^{\text {th }}$ integer. However, particles with large synchrotron amplitudes could see these resonances (and nearby higher order difference resonances) due to high chromaticity.

\section{Beam-beam contribution to chromaticity}

Since chromaticity is important for stability of both incoherent and coherent motion let us consider it in more detail. Fig.2 (left) presents measured antiproton bunch-bybunch chromaticity [5] in store 3678 (average for the three trains). Bare lattice chromaticity on the pbar helix was $C_{x}=10.5, C_{y}=11.5\left(C_{x}=12.5, C_{y}=10.5\right.$ on the proton helix) so there was a large contribution from beam-beam interactions.

Both head-on and long-range interactions contribute to the chromaticity: the head-on interactions owing to $\beta$ function modulation

$$
C_{x, y}^{(\mathrm{HO})}=\Delta Q_{x, y}^{(\mathrm{HO})} W_{x, y}, \quad W_{x, y}=\frac{1}{\beta_{x, y}} \frac{\partial \beta_{x, y}}{\partial \delta_{p}},
$$



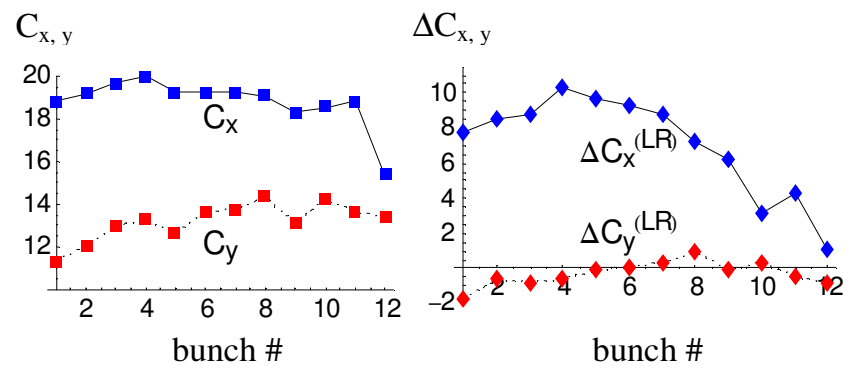

Figure 2. Measured antiproton chromaticity (left) and calculated long-range contribution for particles with small amplitudes (right) vs. bunch number in a train.

whereas the long-range interactions mainly owing to modulation of the beam separation $d_{x, y}[6]$ :

$$
C_{x, y}^{(\mathrm{LR})}=\left(D_{x} \frac{\partial}{\partial d_{x}}+D_{y} \frac{\partial}{\partial d_{y}}\right) \Delta Q_{x, y}^{(\mathrm{LR})} \sim \frac{2 D_{x}}{d_{\text {full }}} \Delta Q_{x, y}^{(\mathrm{LR})},
$$

$D_{x, y}$ being dispersion functions. Both contributions can be a few units large. The head-on chromaticity is about the same for all bunches, whereas the long-range contribution varies from bunch to bunch.

Fig.2 (right) shows analytically calculated long-range chromaticity for antiprotons with small amplitudes; one can see some resemblance with the measurement data.

\section{Emittance growth}

The proximity of the Tevatron working point to the $5^{\text {th }}$ order resonances has an adverse effect on the pbar emittance at the start of HEP. Fig.3 shows increase in the vertical emittance of antiprotons along one bunch train. Dependence on bunch position ("scallops") is a clear signature of long-range interactions; it correlates with bunch-by-bunch tunes and chromaticities.

However, there is a puzzle: usually "scallops" are more pronounced in the vertical plane [1] despite smaller vertical chromaticity.

A tentative explanation of this apparent contradiction is based on the proximity of tunes in all pbar bunches except the last one to the main diagonal. Usually the horizontal emittance is larger so the coupling redistributes energy from the horizontal to the vertical plane.

But the total increase is due to the $5^{\text {th }}$ integer; to avoid it a few measures were proposed (besides reduction in chromaticity): compression of the pbar tunespread with the help of nonlinear electron lenses, moving to another

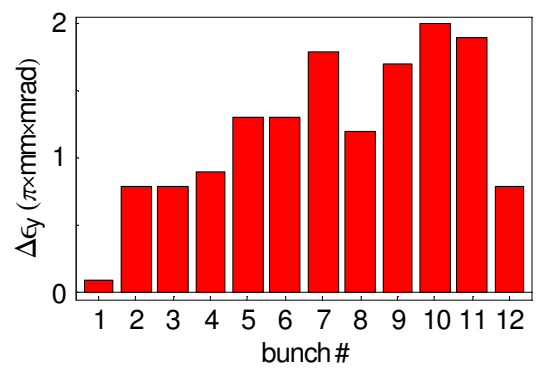

Figure 3. Antiproton vertical emittance growth in $15 \mathrm{~min}$ of collisions (store \#3554, 06/02/04). Only one train shown due to 3 -fold symmetry.

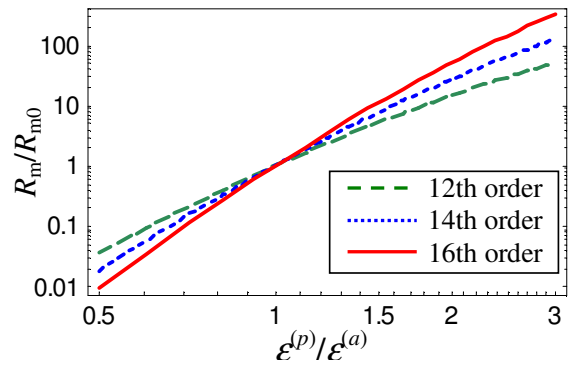

Figure 4. Dependence of head-on resonance driving term at $3 \sigma$ betatron amplitude on the ratio of emittances of the probe and acting beams.

working point (e.g. that used in SPS).

Let us note that antiprotons with large betatron amplitudes do not see the $5^{\text {th }}$ order resonances (Fig.1), therefore these resonances are not the immediate cause of particle losses.

\section{Particle losses}

Beam-beam induced losses pose a serious problem: they create high backgrounds at detectors (especially at the start of HEP) and reduce luminosity lifetime. Surprisingly, the strong beam in the weak-strong case may suffer higher losses $[1,2]$.

This can be explained by smaller emittance of the antiproton beam in both SPS and Tevatron since the strength of high-order resonances excited in head-on interactions is very sensitive to the emittance ratio.

Fig.4 shows dependence of $12^{\text {th }}, 14^{\text {th }}$ and $16^{\text {th }}$ order resonance driving terms (RDTs) on this ratio. In the limit $\varepsilon_{p} / \varepsilon_{a}<<1 R_{m} \sim\left(\varepsilon_{p} / \varepsilon_{a}\right)^{m / 2}$, but at $\varepsilon_{p} / \varepsilon_{a}>1$ the dependence is weaker.

The so-called quasi-linear diffusion rate [7] is proportional to the RDT squared:

$$
D_{Q L}=2 \pi R_{\underline{m}}^{2} / v_{M}
$$

$\nu_{M}=\left(m_{x} C_{x}+m_{y} C_{y}\right) \delta_{p 0}$ being the resonance tune modulation by synchrotron oscillations, so one would expect it to scale as $\left(\varepsilon_{p} / \varepsilon_{a}\right)^{m}$.

Observations however do not confirm such strong dependence. Fig. 5 shows proton background rate in SPS

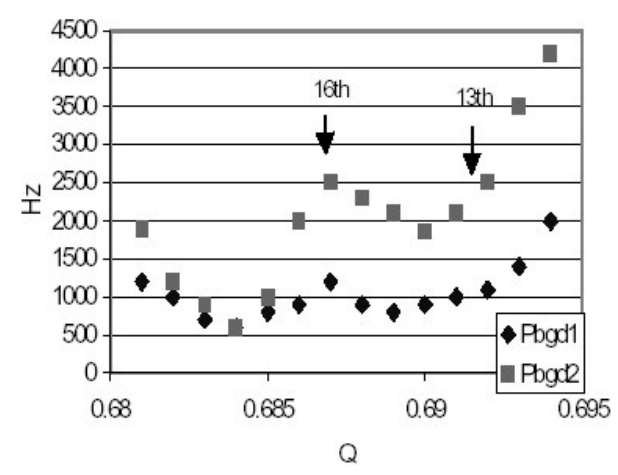

Figure 5. Proton background in SPS vs. horizontal tune before (pbgd1) and after (pbgd2) the pbar emittance was reduced by $30 \%$ [2]. 


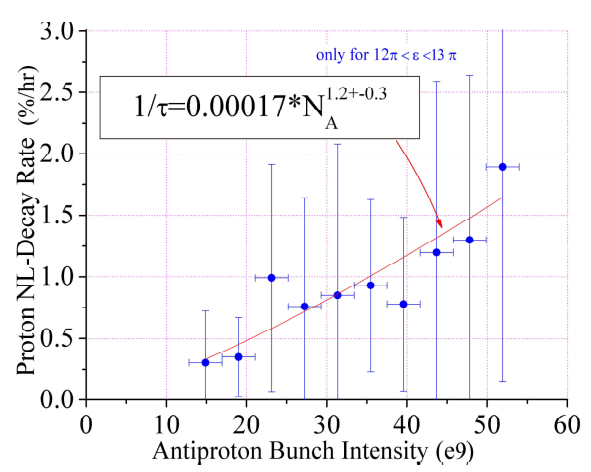

Figure 6. Non-luminous loss rate from proton bunches in Tevatron vs intensity of antiproton bunches they collide head-on with. Data from 51 stores in Dec. 2004 - Feb. 2005. (Courtesy of V.Shiltsev).

(induced by the $16^{\text {th }}$ order resonances) at two values of the pbar emittance [2]. From the plot one can infer scaling $\left(\varepsilon_{p} / \varepsilon_{a}\right)^{2}$. The same law was obtained for proton losses in Tevatron induced by the $12^{\text {th }}$ order resonances [1].

Another contradiction with the model of overlapping synchrotron satellites [7] can be seen in the loss rate dependence on the acting (pbar) beam intensity. Fig. 6 shows that in Tevatron for a given emittance ratio $(\approx 1.7$ in the plot) the losses grow as $N_{a}$, whereas Eq. 3 suggests scaling as $N_{a}^{2}$.

Even more important is the absence of a threshold intensity: this means that the diffusion is caused likely by another mechanism than the resonance overlap.

Large statistical errors do not permit to make definite conclusions, the more so that the HERA observations indicate a stronger dependence on intensity of the opposing electron beam [8]. The work in this direction should be continued in order to understand the scaling law of the losses.

\section{COHERENT BEAM-BEAM EFFECTS}

Beam-beam interaction affects the coherent motion in a number of ways. First of all, it introduces incoherent tunespread which in principle can provide Landau damping of coherent oscillations, but at the same time

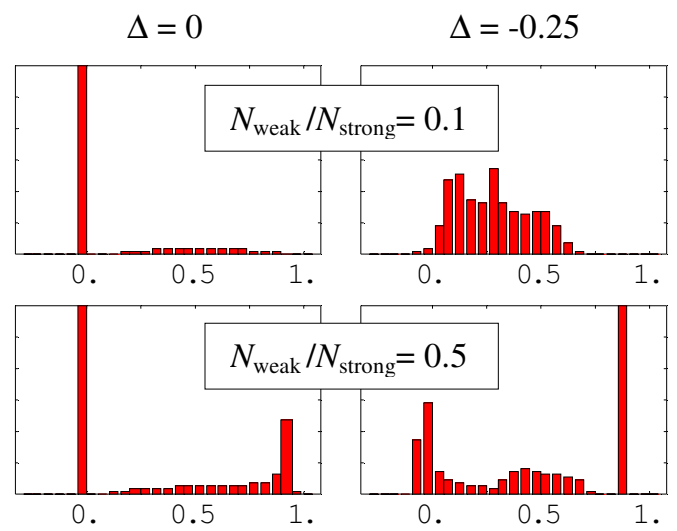

Figure 7. Spectral density of coherent oscillations (arbitrary units) as function of $\Delta Q_{\text {coherent }} / \xi_{\text {weak }}$ at two values of tunesplit $\Delta=\left(Q_{\text {weak }}-Q_{\text {strong }}\right) / 2 \xi_{\text {weak }}$
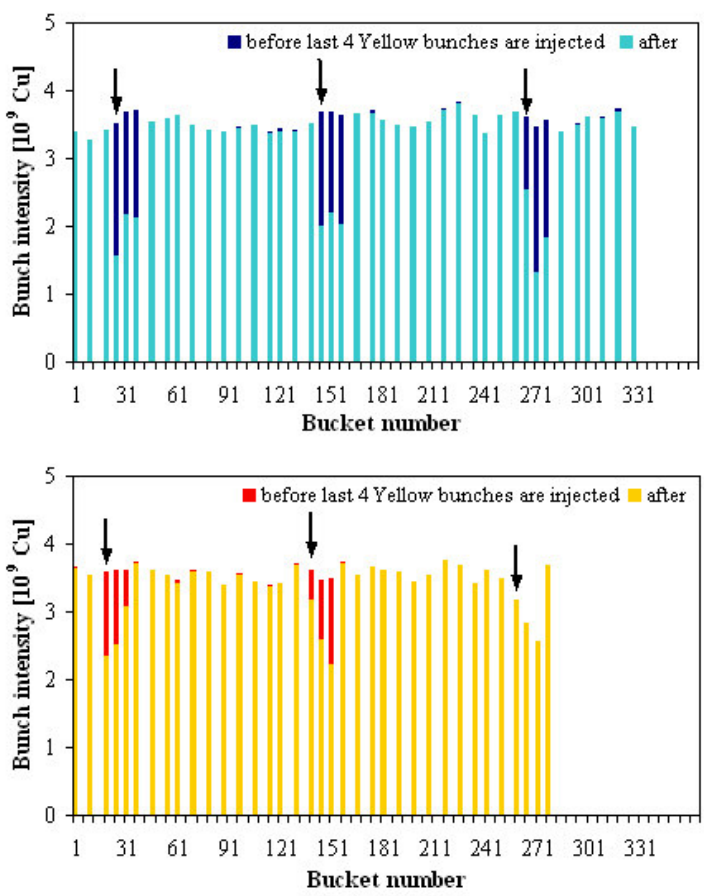

Figure 8. Bunch-by-bunch distribution of losses in the result of instability of coherent beam-beam oscillations during injection in RHIC [4] (courtesy of W.Fischer).

beam-beam interaction can shift the coherent tune out of the incoherent tunespread switching off this damping mechanism completely and leaving the beams exposed to an impedance-driven instability [9].

Also, beam-beam interaction can drive specific coherent resonances. The coherent beam-beam resonances were observed in $e^{+} e^{-}$machines, but in hadron colliders they were not seen since the working point is usually sufficiently far away from low-order resonances.

\section{Coherent modes in head-on colliding beams}

Coherent modes are usually thought of as rigid-bunch oscillations, though due to nonlinearity such motion is not possible with exception for the $\Sigma$-mode when the beams oscillate in phase (for this the bare lattice tunes should be equal). Beam-beam interaction does not affect this mode at all.

As concerns the $\pi$-mode (when the beams oscillate out of phase), in real (soft) bunches there is an infinite number of such modes: continuum with tunes distributed over the same range as incoherent tunes, and possibly a few discrete modes with tunes out of this range [10].

The coherence of beam-beam oscillations is characterized by the Yokoya factor

$$
Y=\Delta Q_{\text {coherent }} / \max \left(\Delta Q_{\text {incoherent }}\right),
$$

where $Q_{\text {coherent }}$ is the $\pi$-mode tuneshift.

In head-on colliding round beams of equal intensity $Y=1.214$. Theory predicts a number of stabilizing factors, such as tunesplit, unequal intensities, synchrotron sidebands overlap [10].

Fig. 7 presents the Vlasov perturbation theory prediction for spectra of oscillations in the strong bunch at 


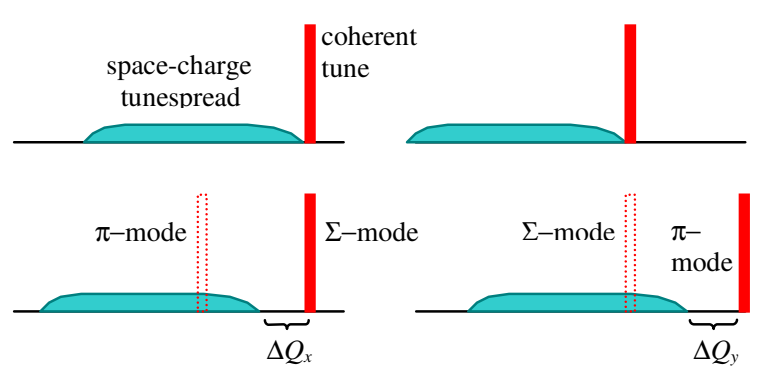

Figure 9. Schematic of the effect of long-range interaction of vertically separated beams on the horizontal (left) and vertical (right) oscillations.

the indicated values of intensity ratio and (half) split in bare lattice tunes $\left(Q_{\text {weak }}-Q_{\text {strong }}\right) / 2$, normalized by the beam-beam parameter for the weak beam, $\xi_{\text {weak }}$.

At small value of intensity ratio the tunesplit completely suppresses coherent oscillations, which may explain absence of instability at zero chromaticity in the Tevatron Run I [11]. With increasing intensity ratio the tunesplit becomes less efficient, in its presence the $\pi$ mode emerges from the incoherent tunespread even earlier than in its absence.

\section{Long-range interactions}

For long-range interactions the Yokoya factor is large, $Y \approx 2$, which increases the danger of instability.

Instability of coupled oscillations of two $\mathrm{Cu}$ beams was recently observed at RHIC at injection energy [4]. Longrange interaction at six crossing points couples sets by three bunches in each beam, some of these sets may go unstable while others do not. Fig. 8 shows bunch intensities before and after the last group of bunches was injected into the Yellow ring sparking the instability.

The tentative explanation of this effect is based on the shift of coherent tunes by beam-beam interaction with respect to the incoherent tunespread produced by spacecharge forces (Fig. 9).

Since the beams are vertically separated the beambeam tuneshift is negative in the horizontal plane and positive in the vertical plane, whereas the space-charge tuneshift is negative in both planes. It is easy to see that by shifting the horizontal incoherent tunes further down the beam-beam interaction leaves undamped the horizontal $\Sigma$-mode (which is not shifted at all), in the vertical plane the $\pi$-mode is shifted up by $Y \approx 2$ times as much as the incoherent tunes and also may become unstable (Fig. 9).

The instability can be suppressed with the help of tunesplit (to restore Landau damping) or chromaticity increase (to enhance the head-tail damping) [4].

\section{Multibunch modes}

In machines like Tevatron and LHC where bunches experience multiple long-range interactions such measures as splitting the tunes may be not enough to ensure stability even with very low intensity of one of the beams.

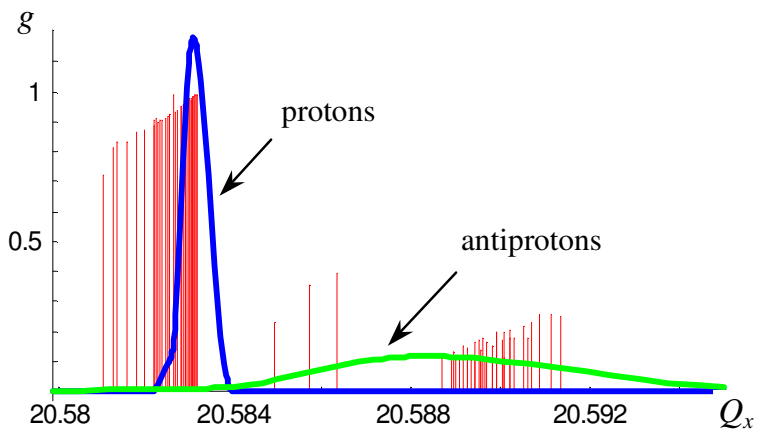

Figure 10. Growth rates of multibunch modes in Tevatron (red lines) and spectral functions of the proton (blue) and antiproton (green) beams.

Fig.10 presents spectrum of multibunch modes of horizontal oscillations of $36 \times 36$ bunches in Tevatron calculated in the rigid-bunch approximation with the bare lattice tunes $Q_{x}{ }^{(\mathrm{a})}=20.577, Q_{x}{ }^{(\mathrm{p})}=20.582$ and the intensities per bunch $N_{p}=2 \cdot 10^{11}, N_{a}=2 \cdot 10^{10}$. The ordinate gives the mode growth rates due to effect of the wall impedances which were modeled in calculations by assigning a small imaginary part to the bare lattice tunes proportional to the bunch intensity (equals unity for protons in the plot).

One can see that multiple long-range interactions (70 for each bunch) can shift the $\Sigma$-like mode tunes far from the proton incoherent tunespread (the blue curve shows the spectral function of all 36 proton bunches), however their growth rates are almost as high as for the proton beam alone.

Though the tunes of all modes lie within the antiproton incoherent tune distribution (the green curve shows the spectral function of antiprotons) the pbar intensity may be not sufficient to suppress the instability.

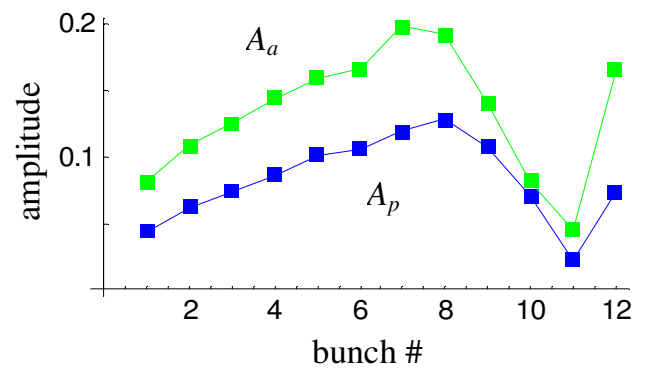

Figure 11. Amplitudes of oscillations of the proton (blue) and antiproton (green) bunches vs. the bunch position in the train for the second rigid-bunch mode from Fig.10.

It is interesting that though the growth rate of these modes is inherited from the proton beam, the antiprotons participate in oscillations with larger amplitude. Fig.11 shows bunch-by-bunch amplitudes for the second mode (counted from the left in Fig.10).

These results can explain observations of coherent instability of colliding beams in Tevatron at low values of chromaticity. The last such experiment was performed in the end of store 4105 on $04 / 21 / 05$ and resulted in a quench. 

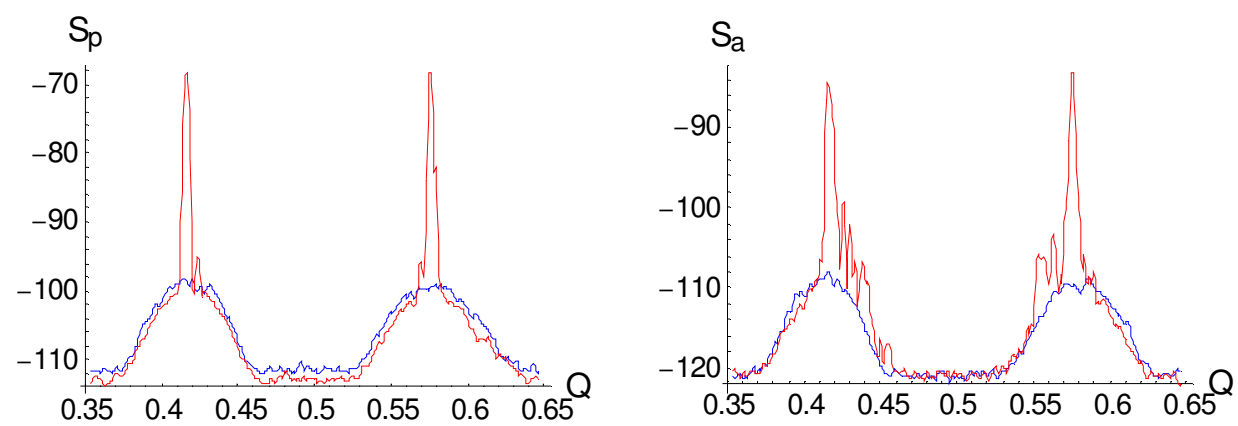

Figure 12. Schottky spectra in the proton (left) and antiproton (right) beams before the onset of instability (blue) and just before the quench (red) during the 04/21/05 studies at Tevatron (data provided by A.Jansson)

First the vertical chromaticity was lowered from 10.5 to 2.5 units which caused some insignificant increase in the Schottky power, but when the horizontal chromaticity was lowered from 7 to 1.5 units the beams went unstable.

The instability was rather slow which permitted to record bunch-by-bunch losses prior to the quench. They indicate that it was a multibunch instability with both beams participating, the quench being caused by antiprotons.

Also it was possible to register the Schottky power from each beam $(1.7 \mathrm{GHz}$ monitor has such capability [5]) as the instability evolved (Fig. 12). From the difference in the power and the intensity ratio $N_{a} / N_{p}=0.1$ it follows that pbars had a factor of 4 larger amplitude, very much in line with the rigid-bunch model predictions.

The rigid-bunch approximation does not provide direct information on Landau damping, it could only be inferred from the value of the beam spectral function at the mode tune. To simulate multibunch modes beyond this approximation presents a real challenge even for the most powerful computers. The work on such codes is going on at Fermilab and CERN [12].

In conclusion of this section let us note that instability of coherent beam-beam oscillations was one of the factors limiting the ISR performance [13]. The instability was most likely impedance-driven, the role of the beam-beam interaction was to modify the spectrum of coherent oscillations in such a way that the frequency of the most unstable mode occured outside the transverse damper range, in some analogy with the effects discussed above.

\section{SUMMARY}

There is at least qualitative understanding of the beambeam effect in hadron colliders, the theory does reflect the reality. However not all of the detail is reproduced quantitatively, the work on refining the existing simulation codes and development of new ones (especially for the coherent beam-beam effect) should be continued.

\section{ACKNOWLEDGEMENTS}

The author is grateful to V. Lebedev, V. Shiltsev (FNAL), W. Fischer (BNL), J.-P. Koutchouk and W. Herr (CERN) for stimulating discussions.

\section{REFERENCES}

[1] V.Shiltsev et al., "Beam-Beam Effects in the Tevatron Run II", this conference.

[2] K.Cornelis, "Beam-Beam Effects in the SPS", LHC99, Geneva, April 1999, p.2 (CERN-SL-99-039 AP).

[3] W.Fischer et al., PAC'03, Portland, May 2003, p.135.

[4] W.Fischer et al., this conference.

[5] A.Jansson, P.Lebrun, R.Pasquinelli, EPAC'04,

Lucerne, Switzerland, July 2004, p.2777.

[6] Y.Alexahin, FERMILAB-TM-2148 (2001).

[7] A.L.Gerasimov, F.M.Izrailev, J.L.Tennyson, AIP Conf. Proceedings 153, v. I, p. 474 (1985).

[8] M.Bieler et al., LHC99, Geneva, April 1999, p.12 (CERN-SL-99-039 AP).

[9] J.Gareyte, in: Proc. $3^{\text {rd }}$ Advanced ICFA Beam Dynamics Workshop on Beam-Beam Effects in Circular Colliders, Novosibirsk, 1989, pp.135-139.

[10] Y.Alexahin, NIM A480 (2002) 253-288.

[11] V.Bharadwaj et al., FERMILAB-TM-1970 (1996).

[12] T.Pieloni and W.Herr, this conference.

[13] J.-P.Koutchouk, unpublished. 